\title{
CHARACTERIZATION OF INTERFACES IN SEMIMAGNETIC QUANTUM WELLS
}

\author{
G. SchmitT, B. Kuhn-Heinrich, U. Zehnder, W. Ossau, T. Litz, \\ A. WAag AND G. LANDWEHR \\ Physikalisches Institut der Universität Würzburg \\ Am Hubland, 97074 Würzburg, Germany
}

\begin{abstract}
The interfaces between nonmagnetic CdTe quantum wells and semimagnetic barriers of $\mathrm{Cd}_{1-x} \mathrm{Mn}_{x} \mathrm{Te}$ were investigated for several well widths by low temperature photoluminescence and photoluminescence excitation spectroscopy. Specially designed $\mathrm{Cd}_{1-x} \mathrm{Mn}_{x} \mathrm{Te} / \mathrm{CdTe} / \mathrm{Cd}_{1-y} \mathrm{Mg}_{y}$ Te structures enable us to distinguish the quality of the semimagnetic normal and inverted interfaces. The normal interface shows to have a better structural quality than the inverted interface.
\end{abstract}

PACS numbers: 78.55.Et, 78.66.-w

The overall quality of complex heterostructures is mainly influenced by the quality of the interfaces between the different layers of these structures. Improving our knowledge about semiconductor-semiconductor interfaces is, therefore, important for the development of new electronic and photonic devices. Most studies carried out so far have been based on the analysis of the linewidth of the excitonic luminescence [1]. Heterostructures containing diluted magnetic semicon, ductors (DMS) offer another possible access to characterize interfaces [2-3]. The exchange interaction of free carriers (excitons) with the Mn spins of the barrier causes a large Zeeman splitting (ZS), that is especially sensitive to the profile of the manganese contents at the interface region [3]. As the ZS is not sensitive to nonmagnetic materials, we studied $\mathrm{Cd}_{1-x} \mathrm{Mn}_{x} \mathrm{Te} / \mathrm{CdTe} / \mathrm{Cd}_{1-y} \mathrm{Mg}_{y} \mathrm{Te}$ structures with DMS on one barrier side only. Due to its small lattice mismatch of $1 \%$ [4] only, MgTe has recently shown to be an ideal barrier material to CdTe based heterostructures [5]. The use of $\mathrm{MgTe}$ as a nonmagnetic counterpart to MnTe enables us to distinguish the different interfaces of a quantum well (QW) and to investigate the normal (ternary on binary material) and the inverted (binary on ternary material) semimagnetic interfaces separately.

Figure 1a shows the band structures of the studied samples, which consist of a set of electronically uncoupled $\mathrm{CdTe}$ QWs with well widths ranging from $12 \AA$ to $150 \AA$ separated by alternating $500 \AA$ wide barrier layers of $\mathrm{Cd}_{1-x} \mathrm{Mn}_{x} \mathrm{Te}$ and $\mathrm{Cd}_{1-y} \mathrm{Mg}_{y} \mathrm{Te}$. Instead of the recently applied system $(\mathrm{CdZn}) \mathrm{Te} / \mathrm{CdTe} /(\mathrm{CdMn}) \mathrm{Te}$ [3] the nonmagnetic ( $\mathrm{CdMg}) \mathrm{Te}$ barriers are as high as the semimagnetic (CdMn)Te 


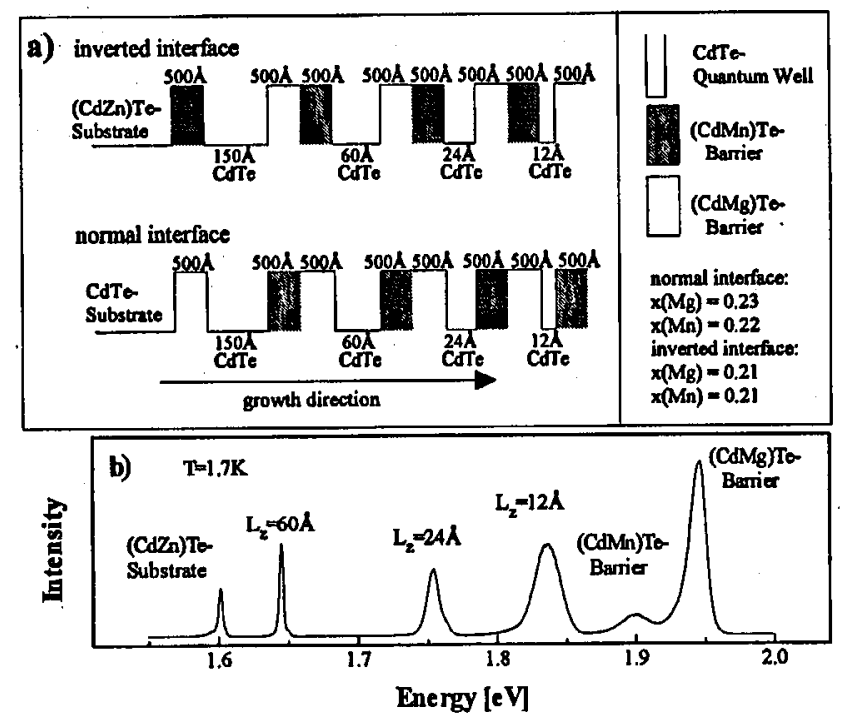

Fig. 1. (a) Structures of the investigated samples with one semimagnetic barrier at the normal or inverted interface only. (b) $\mathrm{PL}$ spectra of the $\mathrm{Cd}_{0.79} \mathrm{Mn}_{0.21} \mathrm{Te} /$ $\mathrm{CdTe} / \mathrm{Cd}_{0.79} \mathrm{Mg}_{0.21} \mathrm{Te}$ heterostructure with the semimagnetic layer at the inverted interface at $T=1.7 \mathrm{~K}$.

barriers, resulting in an unstrained heterostructure with symmetrical band alignment on both sides of the QW. The difference of the structures lies in the arrangement of the barriers, only. Figure $1 \mathrm{~b}$ displays the photoluminescence spectra at $1.7 \mathrm{~K}$ of the structure that has the semimagnetic barrier layer at the inverted interface. The linewidths range from $3 \mathrm{meV}$ for the $\mathrm{QW}$ with $L_{z}=60 \AA$ up to $22 \mathrm{meV}$ for the $12 \AA \mathrm{QW}$. These results are similar to the linewidths observed for CdTe QWs with either (CdMn)Te or (CdMg)Te barrier layers on both sides of the QWs [5]. The structure with the semimagnetic barrier layer at the normal interface shows similar linewidths as that with the inverted semimagnetic interface. This means that the overall structural quality of the two different samples is not determined by the arrangement of the barrier layers. A pure analysis of the linewidth of the excitonic luminescence, however, always means an averaging over the effects both interfaces of a QW contribute to the broadening of the linewidth.

A distinction of these two different interfaces is possible by characterizing the interfaces through the Zeeman effect. Sensitive to semimagnetic material only, the $\mathrm{ZS}$ provides information about the interface quality of the inverted and the normal semimagnetic interface separately. With photoluminescence excitation we measured the energy of the heavy hole exciton under right- and left-circularly polarized excitation as a function of the magnetic field in Faraday configuration. In Fig. 2a we show, for both types of interfaces, the total ZS obtained for the QWs with $L_{z}=60 \AA$. Although in both samples DMS makes up one barrier side only, the obtained ZSs are large compared with structures having two semimagnetic barriers. Two physical mechanisms are mainly responsible for this enhancement of 

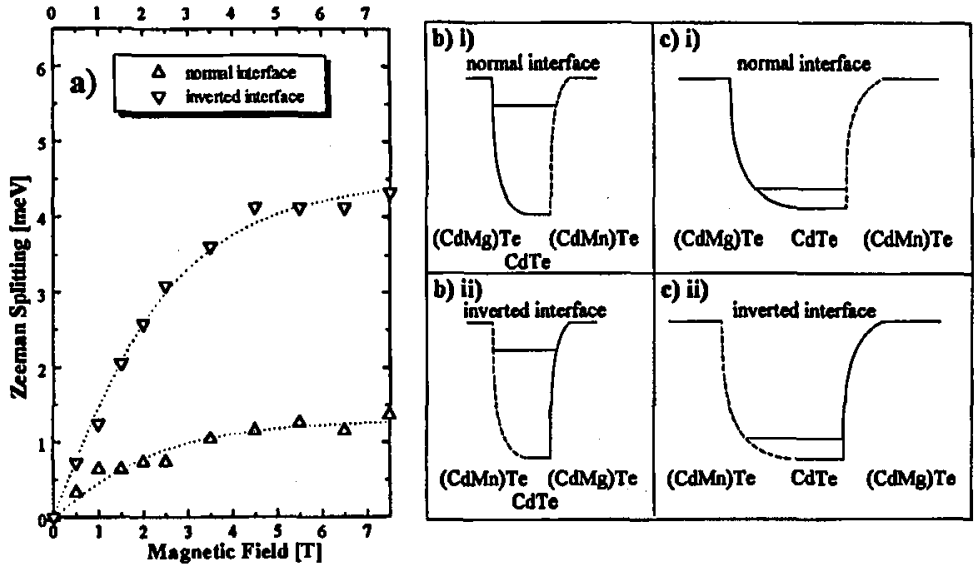

Fig. 2. (a) Comparison of the Zeeman splittings of the quantum wells with $L_{z}=$ $60 \AA$ for the semimagnetic normal and inverted interface. (b) Narrow quantum wells with exponential Mn concentration profiles at the normal (i) or inverted (ii) interface. The dashed areas indicate the semimagnetic interfaces. (c) Wide quantum wells with exponential Mn concentration profiles at the normal (i) or inverted (ii) interface. The dashed areas indicate the semimagnetic interfaces.

TABLE

Total Zeeman splittings of inverted and normal interface for several well widths at $B=7.5 \mathrm{~T}$, ratio $R$ of the total Zeeman splittings at the inverted interface to the total Zeeman splittings at the normal interface.

\begin{tabular}{c|c|c|c}
\hline \hline \multirow{3}{*}{$\begin{array}{c}\text { Well width } \\
{[\AA]}\end{array}$} & Inverted interface & Normal interface & \\
\cline { 2 - 3 } & Zeeman splitting & Zeeman splitting & Ratio \\
at $B=7.5 \mathrm{~T}[\mathrm{meV}]$ & at $B=7.5 \mathrm{~T}[\mathrm{meV}]$ & $R$ \\
\hline 60 & 4.3 & 1.3 & $3.5 \pm 0.5$ \\
24 & 29.3 & 29.0 & $1.0 \pm 0.2$ \\
12 & 59.8 & 116.1 & $0.5 \pm 0.1$
\end{tabular}

the ZSs of the QWs: interface mixing and intrinsic magnetic interface effects [3]. The latter means that the Mn spins sitting at an ideal CdTe interface have less Mn neighbors than those inside the barrier and exhibit, therefore, an enhanced paramagnetism. This effect should be the same for both types of interfaces and cannot explain the difference of the ZSs we see in Fig. 2a. The second source of dilution of the magnetic ions is the segregation of $\mathrm{Mn}$ into the QW during the growth. As segregation of $\mathrm{Mn}$ ions into the $\mathrm{QW}$ is more likely at the inverted interface, the broadening of the inverted interface region is more pronounced. Therefore, the ZS of the inverted interface is larger than that observed at the normal interface (see Fig. 2a). The results of the total ZS at $B=7.5 \mathrm{~T}$ for all investigated well widths are summarized in Table. The ratio $R$ of the total ZSs at the inverted interface to 
the total ZSs at the normal interface (see Table) reveals an unexpected dependence on the well width. It decreases from a value of about 3.5:1 (for $L_{z}=60 \AA$ ) to 1:1 (for $L_{z}=24 \AA$ ) and finally to a value of $1: 2$ (for $L_{z}=12 \AA$ ). Assuming a symmetric concentration profile of the $\mathrm{Mn}$ concentration at the interface region, e.g. an error function profile produced by diffusion processes, this behavior cannot be explained [3]. We have to assume a strongly asymmetric $\mathrm{Mn}$ concentration profile, e.g. an exponential profile induced by a segregation process. In narrow QWs the excitonic wave function penetrates the barrier more than in broader QWs. The asymmetry of the interface profile has the effect that in the barrier region that is additionally probed by the high-energy subband of narrow QWs the gradient of the $\mathrm{Mn}$ concentration is weaker at the normal interface than at the inverted interface (see Fig. 2b). Due to this spread-out area of dilution of the Mn ions paramagnetism is enhanced at the normal interface. In broad QWs (see Fig. 2c), where mainly the well region is probed by a low-energy subband, the gradient is smaller at the inverted interface resulting in the observed bigger ZS of this structure.

In conclusion, we have demonstrated that heterostructures with DMS on one barrier side only are apt to distinguish the inverted and the normal interfaces via an analysis of the ZS. We have found for broad well widths a better structural quality of the normal interface, while the behavior of the ZS in narrow quantum wells gives hints for an asymmetric Mn concentration profile at the interfaces.

The authors wish to acknowledge helpful discussions with J. Gaj and the financial support by the Deutsche Forschungsgemeinschaft through SFB 410.

\section{References}

[1] M.A. Herrmann, D. Bimberg, J. Christen, J. Appl. Phys. 70, R1 (1991).

[2] J.K. Furdyna, J. Appl. Phys. 64, R29 (1988).

[3] J.A. Gaj, W. Grieshaber, C. Bodin-Deshayes, J. Cibert, G. Feuillet, Y. Merle d'Aubigné, A. Wasiela, Phys. Rev. B. 50, 5512 (1994).

[4] A. Waag, F. Fischer, T. Litz, B. Kuhn-Heinrich, U. Zehnder, W. Ossau, W. Spahn, H. Heinke, G. Landwehr, J. Cryst. Growth 138, 155 (1994).

[5] W. Ossau, U. Zehnder, B. Kuhn-Heinrich, A. Waag, T. Litz, G. Landwehr, Superlattices Microstruct. 6, 5 (1994). 